\title{
Estrogen Receptor Polymorphism Predicts the Onset of Natural and Surgical Menopause*
}

\author{
ANGÉLIQUE E. A. M. WEEL, ANDRÉ G. UITTERLINDEN, IRIS C. D. WESTENDORP, \\ HUIBERT BURGER, STEPHANIE C. E. SCHUIT, ALBERT HOFMAN, \\ THEO J. M. HELMERHORST, JOHANNES P. T. M. VAN LEEUWEN, \\ AND HUIBERT A. P. POLS, \\ Departments of Internal Medicine III (A.E.A.M.W., A.G.U., H.B., S.C.E.S., J.P.T.M.v.L., H.A.P.P.), \\ Epidemiology and Biostatistics (A.E.A.M.W., A.G.U., I.C.D.W., H.B., A.H., H.A.P.P.), and Obstetrics \\ and Gynecology (T.J.M.H.), Erasmus University Medical School, 3000 DR Rotterdam, The \\ Netherlands
}

\begin{abstract}
Age at menopause and risk of hysterectomy have strong genetic components, but the genes involved remain ill defined. We investigated whether genetic variation at the estrogen receptor $(\mathrm{ER})$ gene contributes to the variability in the onset of menopause in 900 postmenopausal women, aged 55-80 yr, of the Rotterdam Study, a population-based cohort study in The Netherlands. Gynecological information was obtained, and if women reported surgical menopause, validation of type and indication of surgery was accomplished by checking medical records. The ER genotypes (PP, $\mathrm{Pp}$, and $\mathrm{pp}$ ) were assessed by PCR using the PvuII endonuclease.

Compared with women carrying the pp genotype, homozygous PP
\end{abstract}

women had a 1.1-yr $(P<0.02)$ earlier onset of menopause. Furthermore, an allele dose effect was observed, corresponding to a 0.5 -yr $(P<0.02)$ earlier onset of menopause per copy of the $\mathrm{P}$ allele. The risk of surgical menopause was 2.4 (95\% confidence interval, 1.5-3.8) times higher for women carrying the PP genotype compared to those in the pp group, with the most prominent effect in women who underwent hysterectomy due to fibroids or menorrhagia.

We conclude that genetic variations of the ER gene are related to the onset of natural menopause and the risk of surgical menopause, especially hysterectomy. ( $J$ Clin Endocrinol Metab 84: 3146-3150, 1999)

candidate in this respect. This member of the family of steroid transcription factors functions as a regulator of the expression of many genes and proteins $(13,14)$, and furthermore, the ER is an important regulator of growth and differentiation in many tissues, including the endometrium (15-18).

The aim of the present study was to identify a genetic determinant of the onset of menopause. We investigated the association between an anonymous intronic PvuII restriction fragment length polymorphism (RFLP) of the ER gene and both the natural and surgical onsets of menopause in a population-based sample of postmenopausal women.

\section{Materials and Methods} pause of $63 \%$ (12). In addition, undergoing hysterectomy before reaching natural menopause, with menorrhagia or fibroids as main indications, showed considerable heritability $(59 \%)$ in the same study.

Several approaches can be followed to identify genes that might contribute to the variation in the onset of menopause, including the analysis of candidate genes. In the estrogen endocrine system the estrogen receptor (ER) is an important

Received February 3, 1999. Revision received April 28, 1999. Accepted May 27, 1999.

Address all correspondence and requests for reprints to: Prof. Dr. H. A. P. Pols, Department of Internal Medicine III, Erasmus University Medical School, P.O. Box 1738, 3000 DR Rotterdam, The Netherlands. E-mail: pols@epib.fgg.eur.nl.

* This work was supported by the Netherlands Organization of Scientific Research (Grant 950-10-618).
All postmenopausal women included in this study were part of a population-based cohort study ( $\mathrm{n}=7983 ; 61.1 \%$ women) of persons aged $55 \mathrm{yr}$ and over, living in a district of Rotterdam, The Netherlands. The objective of the study was to investigate the occurrence of chronic disabling diseases in relation to several potential determinants. Rationale and design have been described previously (19). A total of 10,275 persons, of whom 9,161 (89\%) were living independently, were invited to participate in the study in 1990. Among those living independently, the overall response rate was $77 \%$ for the home interview and $71 \%$ for examination at the research center, where anthropometric characteristics and blood samples were taken. The Rotterdam Study was approved by the medical ethics committee of Erasmus University Medical School, and written informed consent was obtained from each subject.

For the present study we included independently living subjects who were initially part of a large epidemiological study of osteoporosis in which subjects according to the following criteria were excluded: aged $80 \mathrm{yr}$ and over, use of thyroid hormone, use of cytostatics, use of diuretics, and known diabetes mellitus type II. Among the 4478 remaining 
independently living subjects, an age-stratified sample of 1000 women was drawn with balanced numbers $(n=200)$ in 5-yr age categories. DNA samples and menopause data for 900 postmenopausal women were available for the analysis.

During the home interview each woman provided information on her reproductive and gynecological history, including ever use of sex steroids. Natural menopause was defined as menopausal after 12 continuous months of amenorrhea, without gynecological surgery or other procedures that would have stopped menses. In case a gynecological surgical procedure before natural menopause was reported, we validated the date and indication of surgery by checking the medical records of the general practitioner ( $98 \%$ of cases). The health care system in The Netherlands permits this validation, as every individual has her own general practictioner. The general practictioner is the only access to specialist and hospital care and preserves all physician and hospital notes. Age at menopause was defined as age at natural menopause or age at surgical menopause (defined as the age at the date of operation). Surgical procedures were defined as hysterectomy (women with only hysterectomy and women with hysterectomy plus uni- or bilateral oophorectomy), oophorectomy (women with only uni- or bilateral oophorectomy), and unknown type of gynecological surgery. Smoking habits (smoking defined as ever vs. never smoked) and socio-economic status (defined as the highest education level attained; class I-II primary school with/without lower secondary school vs. class III-IV secondary school with/without higher vocational school or university) were assessed by questionnaire. Height and weight were measured at the clinical examination, with the subject in a standing position without shoes.

\section{Genotyping}

The anonymous PvuII RFLP is located in intron $1,0.4 \mathrm{~kb}$ upstream of exon 2 of the ER gene and was assessed by a PCR procedure (20). Briefly, genomic DNA (100 ng) was extracted from peripheral leukocytes and used for PCR amplification in a reaction mixture containing $50 \mathrm{mmol} / \mathrm{L}$ $\mathrm{KCl}, 10 \mathrm{mmol} / \mathrm{L}$ Tris- $\mathrm{HCl}$ (pH 8.3), $1.5 \mathrm{mmol} / \mathrm{L} \mathrm{MgCl}_{2}, 0.2 \mathrm{mmol} / \mathrm{L}$ deoxy-NTP, $150 \mathrm{ng}$ of each primer, and $0.2 \mathrm{U}$ Super Taq polymerase (HT Biotechnology Ltd., Cambridge, UK). The reactions were performed in a DNA thermocycler (mode 480, Perkin Elmer Corp., Foster City, CA) with a cycling protocol of 94,60 , and $72 \mathrm{C}$ for 1 min each for 25 cycles. Ten microliters of PCR products were digested with a PvuII restriction enzyme (Life Technologies, Inc., Breda, The Netherlands) and $2.5 \mu \mathrm{L}$ of a buffer [containing $150 \mathrm{mmol} / \mathrm{L}$ Tris- $\mathrm{HCl}(\mathrm{pH} 7.5), 250 \mathrm{mmol} / \mathrm{L} \mathrm{NaCl}$, and $35 \mathrm{~mm} \mathrm{MgCl}$ ] by incubation for $30 \mathrm{~min}$ at $37 \mathrm{C}$. The digestion products were analyzed by $1.4 \%$ agarose gel electrophoresis in $0.5 \times \mathrm{TBE}$ $\left(1 \times \mathrm{TBE}=89 \mathrm{mmol} / \mathrm{L}\right.$ Tris, $89 \mathrm{mmol} / \mathrm{L}$ boric acid, and $2 \mathrm{mmol} / \mathrm{L} \mathrm{Na}_{2}$ ethylenediamine tetraacetate) for 250 volt hours. Separation patterns were documented by Polaroid photography under UV illumination (302 $\mathrm{nm})$. Genotypes were defined as PP, Pp, or pp. Capital letters denote the absence and lowercase letters the presence of the site for the restriction enzyme PvuII $(\mathrm{P} / \mathrm{p})$. To confirm the accuracy of the genotyping, repeated analysis was performed on 100 randomly selected samples. No discrepancies were found.

\section{Statistical analysis}

One-way ANOVA and $\chi^{2}$ analysis were used to compare anthropometric and environmental variables among the three genotype groups. To account for potential confounders, such as age of menarche, mean number of offspring, smoking, body mass index, socio-economic status, hormone replacement therapy, and use of oral contraceptives, we used multivariate regression models. Subsequently, we calculated the odds ratio [with $95 \%$ confidence interval (CI)] as a measure of the relative risk for occurrence and indication of surgical menopause associated with ER genotype using logistic regression models where women without premenopausal gynecological surgery were the reference group. To visualize the genetic influences on the lifetime risk of hysterectomy, we constructed a cumulative hazard function using the Cox proportional hazard regression model where women without surgery were the reference group.

\section{Results}

The allele frequencies did not deviate from the HardyWeinberg equilibrium, which indicates that no selection has occurred among genotypes. Table 1 shows the general characteristics of the postmenopausal women according to their PvuII genotype. The three genotypes did not differ significantly in age at menarche, use of sex steroids (hormone replacement therapy and oral contraceptives), smoking, body mass index, and socio-economic status. Furthermore, the mean number of offspring and the percentage of women with children did not differ between the three genotypes. Unfortunately, we did not have data on the total number of pregnancies, which gives a better insight into fertility.

However, there was a significant difference in the mean

TABLE 1. Characteristics of 900 women according to their ER PvuII genotype

\begin{tabular}{|c|c|c|c|c|c|}
\hline & \multicolumn{3}{|c|}{ ER PvuII genotype } & \multicolumn{2}{|c|}{$P$ value } \\
\hline & $\mathrm{PP}$ & $\mathrm{Pp}$ & $\mathrm{pp}$ & ANOVA & $\mathrm{PP}$ vs. pp \\
\hline No. $(\%)$ & $205(23)$ & $435(48)$ & $260(29)$ & & \\
\hline Age (yr) & $67.9 \pm 7.0$ & $67.5 \pm 6.9$ & $67.1 \pm 7.1$ & 0.4 & 0.2 \\
\hline Ever use oral contraceptive (\%) & $52(25.4)$ & $106(24.4)$ & $61(23.5)$ & 0.9 & 0.8 \\
\hline $\mathrm{Ht}(\mathrm{cm})$ & $162.5 \pm 5.8$ & $161.5 \pm 6.3$ & $161.7 \pm 7.3$ & 0.2 & 0.2 \\
\hline Wt (kg) & $68.6 \pm 10.9$ & $68.4 \pm 10.0$ & $68.2 \pm 10.6$ & 0.9 & 0.5 \\
\hline BMI $\left(\mathrm{kg} / \mathrm{m}^{2}\right)$ & $26.0 \pm 3.8$ & $26.2 \pm 3.5$ & $26.1 \pm 4.1$ & 0.7 & 0.8 \\
\hline No. of offspring & $2.2 \pm 1.6$ & $2.1 \pm 1.6$ & $2.1 \pm 1.8$ & 0.6 & 0.4 \\
\hline \multicolumn{6}{|l|}{ Offspring } \\
\hline None $(\%)$ & $34(16.6)$ & 85 (19.5) & $55(21.2)$ & & \\
\hline 1 or $2(\%)$ & $93(45.4)$ & $198(45.5)$ & $116(44.6)$ & 0.8 & 0.4 \\
\hline$\geq 2(\%)$ & $78(38.0)$ & $152(34.9)$ & $89(34.2)$ & & \\
\hline Age at menopause (yr) & $48.1 \pm 5.0$ & $48.7 \pm 5.0$ & $49.2 \pm 4.6$ & 0.06 & 0.02 \\
\hline Median & $4 \overline{9}$ & $4 \overline{9}$ & 50 & & \\
\hline
\end{tabular}

${ }^{a}$ Natural menopause defined as onset of menopause without hysterectomy, oophorectomy, or any other procedure that stopped menses.

${ }^{b}$ Age at surgical menopause defined as age at date of hysterectomy and/or oophorectomy. 
age at onset of menopause among the genotypes. The mean and median age at menopause were $1.1 \mathrm{yr}(P<0.02)$ earlier in women with the PP genotype compared to those in the pp genotype group. Furthermore, an allele-dose effect was observed, corresponding to a $0.5-y r(P<0.02)$ earlier onset of menopause per copy of the $\mathrm{P}$ allele. After adjustment for the potential confounders (age at menarche, mean number of offspring, use of sex steroids (HRT and oral contraceptives), smoking, body mass index, and socio-economic status), similar findings were observed.

After excluding women with artificial menopause, the result remained essentially the same, with an earlier onset of natural menopause for the women with the PP genotype. However, the frequency of women with surgical menopause differed among the three genotypes (Table 2). Therefore, we compared the risk of surgical menopause for women by their ER genotype. The prevalence of women with surgical menopause was highest in women with the PP genotype, lower in the heterozygous Pp women, and lowest in women with the pp genotype (Table 2). This overrepresentation was most notable for the procedure hysterectomy. As shown in Table 2 , the overrepresentation of women with surgical menopause among those with the PP genotype corresponded to a significant 2.4 (95\% CI, 1.5-3.8) times higher risk compared to that in women in the reference group with the genotype pp. When we repeated the analysis by type of surgical procedures, we observed the ER genotype-dependent increased risk of surgical menopause to be due to hysterectomy and not oophorectomy. The odds ratios were 1.7 (95\% CI, 1.3-2.2) and 0.7 (95\% CI, 0.4-1.2)/ copy of P allele for hysterectomy and oophorectomy, respectively. Figure 1 shows the lifetime risk of hysterectomy according to the estrogen receptor genotypes as calculated by the Cox proportional hazard function. The PP group had a significantly higher risk of premenopausal hysterectomy, which confirmed the results from the logistic regression analysis.

In Table 3 we stratified the analysis by indication for surgery [validated data not available for 91 of $174(52.3 \%)$ women with surgery]. The predominant indications were menometrorraghia (23\%) and uterus myomatosis (fibroids; $43 \%$ ). Surgical procedures performed for these indications were hysterectomy or hysterectomy plus oophorectomy. There was no oophorectomy solely performed for these indications. We observed that the ER genotype-dependent risk of hysterectomy to be largely due to the higher frequencies of the indications menometrorraghia and uterus myomatosis (fibroids) in the PP genotype group (Table 3). The odds ratios for the allele dose effect were 2.6 (95\% CI, 1.3-5.2) and 1.8 (95\% CI 1.1-2.9)/copy of the P allele for menometrorrhagia and uterus myomatosis, respectively.

\section{Discussion}

In this population-based study we show for the first time that the common allelic variants of the ER gene are associated with both natural and surgical menopause. Moreover, we provide evidence that women with the PP genotype will undergo hysterectomy due to menorrhagia and fibroids more frequently.

There may have been biases that have lead to incorrect effect estimates. First, the self-report of the age of menopause was determined retrospectively, which has been shown to be unreliable in previous studies (21). Nevertheless, it seems unlikely that this recall bias differs between the ER genotypes. Second, although regional differences in the frequency of hysterectomy have been reported (22-23), it is unlikely that this would have influenced the relation between the ER genotype alleles and the risk of surgical menopause in this

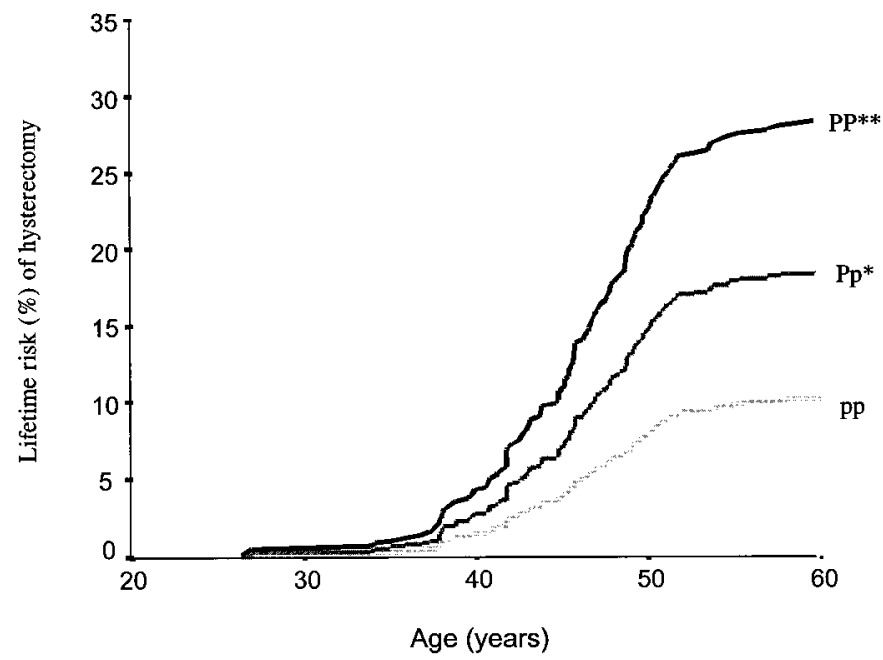

FIG. 1. Lifetime risk (percentage) of premenopausal hysterectomy according to the ER PvuII genotypes. ${ }^{* *}, P<0.001$ (PP genotype $v s$. pp genotype); *, $P=0.01$ (Pp genotype $v s$. pp genotype; according to the Cox proportional hazard model).

TABLE 2. Frequencies and ODDS ratios (95\% CI) for premenopausal gynecological surgical procedures according to ER PvuII genotype

\begin{tabular}{|c|c|c|c|c|}
\hline & \multicolumn{3}{|c|}{ ER PvuII genotype } & \multirow{2}{*}{ Total } \\
\hline & $\mathrm{PP}$ & $\mathrm{Pp}$ & $\mathrm{pp}$ & \\
\hline Surgery $(\%)$ & $55(26.8)$ & $84(19.3)$ & $35(13.5)$ & $174(19.3)$ \\
\hline Hysterectomy $(\%)^{a}$ & $49(23.9)$ & $70(16.1)$ & $24(9.2)$ & $143(15.9)$ \\
\hline Ovariectomy $(\%)$ & $3(1.5)$ & $12(2.8)$ & $10(3.8)$ & $25(2.8)$ \\
\hline Unknown type (\%) & $3(1.5)$ & $2(0.5)$ & $1(0.4)$ & $6(0.7)$ \\
\hline Odds ratio $(95 \% \mathrm{CI})^{b}$ & & & & Allele dose \\
\hline Surgery & $2.4(1.5-3.8)$ & $1.5(1.0-2.4)$ & 1 & $1.5(1.2-1.9)$ \\
\hline Adjusted $^{a}$ & $2.7(1.6-4.3)$ & $1.7(1.1-2.7)$ & 1 & $1.6(1.3-2.1)$ \\
\hline Hysterectomy & $3.1(1.8-5.2)$ & $1.9(1.1-3.1)$ & 1 & $1.7(1.3-2.2)$ \\
\hline Ovariectomy & $0.4(0.1-1.7)$ & $0.8(0.3-1.8)$ & 1 & $0.7(0.4-1.2)$ \\
\hline
\end{tabular}

${ }^{a}$ Adjusted for age, age at menopause, age at menarche, number of offspring, use of sex steroids, smoking habits, SES, and BMI.

${ }^{b}$ The reference group is women without any premenopausal gynecological surgery. 
TABLE 3. Frequency and odds ratio for the indication of surgery according to ER PvuII genotypes

\begin{tabular}{|c|c|c|c|c|}
\hline & \multicolumn{3}{|c|}{ ER PvuII genotype } & \multirow{2}{*}{ Total } \\
\hline & $\mathrm{PP}$ & $\mathrm{Pp}$ & $\mathrm{pp}$ & \\
\hline Surgical procedures & 55 & 84 & 35 & 174 \\
\hline Indication available (\%) & $29(52.7)$ & $43(51.1)$ & $11(31.4)$ & $83(47.7)$ \\
\hline Subjects used for analysis & 179 & 394 & 236 & 809 \\
\hline Menometrorrhagia (\%) & $9(5.0)$ & $8(2.0)$ & $2(0.8)$ & $19(2.3)$ \\
\hline Uterus myomatosis (\%) & $12(6.7)$ & $19(4.8)$ & $5(2.1)$ & $36(4.4)$ \\
\hline Other $(\%)^{a}$ & $8(4.5)$ & $16(4.1)$ & $4(1.7)$ & $28(3.5)$ \\
\hline Odds ratio $(95 \% \mathrm{CI})^{b}$ & & & & Allele dose \\
\hline Menometrorrhagia & $6.7(1.4-31.5)$ & $2.6(0.5-12.2)$ & 1 & $\overline{2.6(1.3-5.2)}$ \\
\hline Uterus myomatosis & $3.6(1.2-10.4)$ & $2.4(0.9-6.6)$ & 1 & $1.8(1.1-2.9)$ \\
\hline Other $^{a}$ & $3.0(0.9-10.1)$ & $2.6(0.8-7.8)$ & 1 & $1.6(0.9-2.8)$ \\
\hline
\end{tabular}

${ }^{a}$ Other includes prolapsed uteri, malignancy, ovarian cyst, or other uncommon diagnosis.

${ }^{b}$ The reference group is women without any premenopausal gynecological surgery.

study. In addition, the allele frequencies of the ER PvuII polymorphism did not differ from frequencies reported previously by others and therefore argue strongly against selection bias in our population (24). Third, although the frequencies of missing data on indication differed among the three genotypes, it is unlikely that the reason for missing these data differed among the ER genotypes. However, in view of the low frequency $(48 \%)$ of validated data on indication for surgery, confirmation of our observations in another population with more extensive clinical and pathological data is needed to provide more robust risk estimates regarding indication. Finally, the ER genotype-dependent effect on end points of menopause may have biased the results, because women included in the study were probably healthier due to the inclusion and exclusion criteria applied. However, as far as we know, no studies have been published about the association of the ER gene and any of these criteria. Moreover, a separate analysis for the total cohort of the Rotterdam study ( $\mathrm{n}=4878$ women) did not show a relation between the inclusion and exclusion criteria and age at menopause (data not shown).

Evidence that genetic factors are related to age at menopause has been observed previously in family and twin studies (10-12). However, until now, only a limited number of genes have been studied in association with the onset of menopause. One study showed that in a single family four woman had an interstitial deletion of the long arm of Xchromosome, which was associated with premature ovarian failure and premature menopause (25). Furthermore, a link between menopause and the galactose-1-phosphate uridyl transferase gene was reported (26). This study, however, had limited generalizability due to population admixture and the small number of subjects examined.

In the present study we observed that common variations of the ER gene are associated with disorders of the uterus. This supports the hypothesis that a common factor is involved in the pathogenesis of abnormal bleeding and uterine fibroids (27). The present observation seems logical due to the fact that the ER has been identified in endometrium, myometrium, and fibroids (15). In addition, estrogens have a direct effect on the uterus, which is emphasized by the fact that ER knockout mice demonstrated lack of uterine response to estrogen treatment compared to wild-type animals (28).

Although it is unclear how the common allelic variations of the ER gene influence the action of the ER for its ligand estradiol, the present study showed an earlier onset of natural menopause and a higher prevalence of hysterectomy due to fibroids and menorrhagia in women carrying the $P$ allele. This suggests a higher responsiveness of this ER gene allele to estrogen, which might affect the biological response and ultimately leads to irregularities in the differentiation and proliferation of endo- and myometrial cells. However, it must be emphasized that it is presently unclear what is the molecular mechanism of the association we here report. Because the PvuII RFLP is an anonymous polymorphism, it seems likely that the $P$ allele is in linkage disequilibrium with a truly causative sequence variation elsewhere in the ER gene or even in another nearby gene. To elucidate the precise molecular mechanism, extensive sequence analysis and functional studies of the ER gene variants are needed.

In conclusion, we have obtained evidence that a common allelic variation in the ER gene is associated with age at menopause as well as hysterectomy. This raises the possibility that genotyping at the polymorphic PvuII ER gene may provide information on susceptibility to uterine disorders leading to early onset of menopause, which eventually might lead to other clinical entities, such as osteoporosis, cardiovascular diseases, and ovarian cancer.

\section{Acknowledgments}

The authors are very grateful to the participants of the Rotterdam study. We also thank Prof. Dr. S. W. J. Lamberts for carefully reading the manuscript, and Dr. C. M. van Duijn for helpful comments and suggestions. Furthermore, we acknowledge Q. Huang, for her technical assistance and all of the participating general practitioners and the many field workers in the research center in Ommoord (Rotterdam, The Netherlands)

\section{References}

1. Gordon T, Kannel WB, Hjortland MC, McNamara PM. 1978 Menopause and coronary heart disease. The Framingham Study. Ann Intern Med. 89:157-161.

2. Seeman E, Cooper ME, Hopper JL, Parkinson E, McKay J, Jerums G. 1988 Effect of early menopause on bone mass in normal women and patients with osteoporosis. Am J Med. 85:213-216.

3. Cramer DW. 1990 Epidemiologic aspects of early menopause and ovarian cancer. Ann NY Acad Sci. 592:363-375.

4. van der Schouw YT, van der Graaf Y, Steyerberg EW, Eijkemans JC, Banga JD. 1996 Age at menopause as a risk factor for cardiovascular mortality. Lancet. 347:714-718. 
5. Brambilla DJ, McKinlay SM. 1990 A prospective study of factors affecting age at menopause [published erratum appears in J Clin Epidemiol 43(5):537]. J Clin Epidemiol. 1989;42:1031-1039.

6. Luoto R, Kaprio J, Uutela A. 1994 Age at natural menopause and sociodemographic status in Finland. Am J Epidemiol. 139:64-76.

7. Stanford JL, Hartge P, Brinton LA, Hoover RN, Brookmeyer R. 1987 Factors influencing the age at natural menopause. J Chronic Dis. 40:995-1002.

8. Whelan EA, Sandler DP, McConnaughey DR, Weinberg CR. 1990 Menstrual and reproductive characteristics and age at natural menopause. Am J Epidemiol. 131:625-632.

9. Midgette AS, Baron JA. 1990 Cigarette smoking and the risk of natural menopause. Epidemiology. 1:474-480.

10. Cramer DW, Xu H, Harlow BL. 1995 Family history as a predictor of early menopause. Fertil Steril. 64:740-745

11. Torgerson DJ, Thomas RE, Reid DM. 1997 Mothers and daughters menopausal ages: is there a link? Eur J Obstet Gynecol Reprod Biol. 74:63-66.

12. Snieder H, MacGregor AJ, Spector TD. 1998 Genes control the cessation of a woman's reproductive life: a twin study of hysterectomy, and age at menopause. J Clin Endocrinol Metab. 83:1875-1880.

13. Evans RM. 1988 The steroid and thyroid hormone receptor superfamily. Science. 240:889-895.

14. Giguere V, Yang N, Segui P, Evans RM. 1988 Identification of a new class of steroid hormone receptors. Nature. 331:91-94.

15. Wilson EA, Yang F, Rees ED. 1980 Estradiol and progesterone binding in uterine leiomyomata and in normal uterine tissues. Obstet Gynecol. 55:20-24.

16. Eriksen EF, Colvard DS, Berg NJ, et al. 1988 Evidence of estrogen receptors in normal human osteoblast-like cells. Science. 241:84-86.

17. Thomas ML, Xu X, Norfleet AM, Watson CS. 1993 The presence of functional estrogen receptors in intestinal epithelial cells. Endocrinology. 132:426-430.

18. Haslam SZ, Shyamala G. 1981 Relative distribution of estrogen and proges- terone receptors among the epithelial, adipose, and connective tissue components of the normal mammary gland. Endocrinology. 108:825-830.

19. Hofman A, Grobbee DE, de Jong PT, van den Ouweland FA, 1991 Determinants of disease and disability in the elderly: the Rotterdam Elderly Study. Eur J Epidemiol. 7:403-422.

20. Yaich L, Dupont WD, Cavener DR, Parl FF. 1992 Analysis of the PvuII restriction fragment-length polymorphism and exon structure of the estrogen receptor gene in breast cancer and peripheral blood. Cancer Res. 52:77-83.

21. Hahn RA, Eaker E, Rolka H. 1997 Reliability of reported age at menopause Am J Epidemiol. 146:771-775.

22. McPherson K, Wennberg JE, Hovind OB, Clifford P. 1982 Small-area variations in the use of common surgical procedures: an international comparison of New England, England, and Norway. N Engl J Med. 307:1310-1314.

23. Mackenbach JP. 1990 Regional differences in the frequency of various common surgical procedures, 1985. Ned Tijdschr Geneeskd. 134:953-957.

24. Gennari L, Becherini L, Masi L, et al. 1998 Vitamin D and estrogen receptor allelic variants in Italian postmenopausal women: evidence of multiple gene contribution to bone mineral density. J Clin Endocrinol Metab. 83:939-944

25. Krauss CM, Turksoy RN, Atkins L, McLaughlin C, Brown LG, Page DC. 1987 Familial premature ovarian failure due to an interstitial deletion of the long arm of the X chromosome. N Engl J Med. 317:125-131.

26. Cramer DW, Harlow BL, Barbieri RL, Ng WG. 1989 Galactose-1-phosphate uridyl transferase activity associated with age at menopause and reproductive history. Fertil Steril. 51:609-615.

27. Deligdish L, Loewenthal M. 1970 Endometrial changes associated with myomata of the uterus. J Clin Pathol. 23:676-680.

28. Korach KS. 1994 Insights from the study of animals lacking functional estrogen receptor. Science. 266:1524-1527.

\section{International Workshop Estrogens and Male Reproduction (EMR) September 23 and 24, 1999 Isola Capo Rizzuto, Calabria, Italy}

This event involves international experts discussing topics dealing with the following topics:

-Aromatase gene expression in male gonad and extragonadal tissue

-Estrogen and male gonad development

-Estrogens, xenoestrogens and male fertility

-Estrogens and growth in males

-Estrogens and bone in males

The Workshop will be preceded by a Meeting on "Osteoporosis and Sex Hormones," September 22, 1999, and will be followed by a Symposium Satellite on "Interrelationships between GH and Sex Hormones," September 25, 1999.

Deadline for early registration at a reduced fee: July 15, 1999. Deadline for abstracts submission: July 15th, 1999.

The abstracts will be published in Molecular and Cellular Endocrinology.

For more information, let's visit on www.prex.it/emr.htm or contact Prex S.r.l., Organizing Secretariat, Viale Monza 137, 20125 Milan, Italy. Tel. +39-02-28311821; Fax +39-02-28311840; E-mail: congressi@prex.it. 